\title{
The Life Satisfaction of Asian Americans: Evidence from the U.S. General Social Survey, 1972 to 2010
}

\author{
Arthur Sakamoto ${ }^{*}$, Chi-Tsun Chiu ${ }^{2}$, Jing $\mathrm{Li}^{3}$, Sharron Xuanren Wang ${ }^{1}$ \\ ${ }^{1}$ Department of Sociology, Texas A\&M University, College Station, USA \\ ${ }^{2}$ Institute of European and American Studies, Academia Sinica, Taiwan \\ ${ }^{3}$ Department of Sociology, Rice University, Houston, USA \\ Email: "asakamoto@tamu.edu,ctchiu@gate.sinica.edu.tw,jingli@rice.edu,xw2683@tamu.edu
}

Received 21 March 2016; accepted 16 April 2016; published 19 April 2016

Copyright (C) 2016 by authors and Scientific Research Publishing Inc.

This work is licensed under the Creative Commons Attribution International License (CC BY). http://creativecommons.org/licenses/by/4.0/

(c) (i) Open Access

\begin{abstract}
We use the U.S. General Social Survey to investigate the overall life satisfaction of Asian Americans. In contrast to most other racial minority groups in the U.S., Asian Americans are advantaged over whites in regard to education and incomes which are known to be correlated with overall life satisfaction. Despite these socioeconomic advantages, Asian Americans express somewhat lower levels of overall life satisfaction than whites. Foreign-born Asian Americans are more likely than whites to be "not too happy" while native-born Asian Americans are less likely than whites to be "very happy." This pattern persists even after controlling for educational attainment and the distribution of household income. The implications of these findings are discussed in regard to the social psychology, assimilation, and demographic characteristics of Asian Americans. Asian Americans are often portrayed as the "Model Minority," but they appear to be somewhat less happy with what they have.
\end{abstract}

\section{Keywords}

Happiness, Asian Americans, U.S. General Social Survey

\section{Introduction}

\subsection{Understanding Overall Life Satisfaction among Asian Americans}

Among other factors, socioeconomic circumstances are consistently associated with self-reported levels of over-

${ }^{*}$ Corresponding author.

How to cite this paper: Sakamoto, A., Chiu, C.-T., Li, J., \& Wang, S. X. (2016). The Life Satisfaction of Asian Americans: Evidence from the U.S. General Social Survey, 1972 to 2010. Sociology Mind, 6, 40-52.

http://dx.doi.org/10.4236/sm.2016.62003 
all life satisfaction (Easterlin, 2001; Blanchflower \& Oswald, 2004). Persons with greater household incomes are more likely to report being "very happy" in cross-sectional social surveys (Clark, Frijters, \& Shields, 2008). In longitudinal surveys, persons who experience a positive change in their income are more likely to report becoming happier (Ravallion \& Lokshin, 2002). While socioeconomic factors are not the only variables affecting reported life satisfaction (Argyle, 1999), prior research consistently reveals net effects of education and income not only in the U.S. but in a variety of other societies including those with developing economies (Graham, 2009).

Understanding the nature of the relationship between socioeconomic status and overall life satisfaction is also pertinent to the study of racial and ethnic minorities in the U.S. who typically have different (usually lower) average levels of education and income (Sakamoto, Goyette, \& Kim, 2009). In regard to African Americans, prior research finds that this minority group is considerably less likely than whites to report a high level of happiness (Campbell, Converse, \& Rodgers, 1975; Blanchflower \& Oswald, 2004; Graham, 2009). This negative racial effect appears to be partly—-though not entirely—mediated by education and income. According to one expert in the field of happiness studies, the "lower happiness of ethnic minorities is mainly due to their lower incomes, education, and job status” (Argyle, 1999: p. 362).

The purpose of this paper is to explore the sources of overall life satisfaction among Asian Americans as a demographic group. This general research objective is motivated by the fact that Asian Americans have apparently not been considered in prior demographic studies of this sort. Asian Americans are currently the fastest growing minority in the U.S. (Pew Research Center, 2013) and research on them lags behind research on other racial and ethnic minorities in America (Sakamoto, Goyette, \& Kim, 2009).

Our primary theoretical focus is on elucidating the roles of education and income in affecting overall life satisfaction (which we hereafter also refer to as "happiness") among Asian Americans. This concern is practically justified because these two variables are directly measured in our data along with an indicator of happiness as well as demographic covariates. While our discussion of the results draws upon considerations of culture and psychology, we do not dwell on these interpretations at length because culture and psychology are too complex to be adequately measured by any of the variables available in our data set. Our broader interpretations are therefore inherently speculative. Our main contribution is to provide systematic information about measured socioeconomic variables using a nationally representative data set (in contrast to ad hoc convenience samples) that allows us to generalize about Asian Americans as an overall demographic group in American society during this time period.

The effects of education and income are also theoretically notable in our study because Asian Americans are the one case of a racial minority that actually has higher average levels of socioeconomic status than whites (Sakamoto, Goyette, \& Kim, 2009). Argyle (1999: p. 362) refers to the "lower incomes, education, and job status" of "minorities" (quoted above) and this description generally applies to African Americans, Hispanics, and Native Americans but not to Asian Americans. In contrast to prior research, our investigation focuses on the case of Asian Americans, but we hope that a byproduct of our analysis is the promotion of a broader perspective on the underlying mechanisms associated with the positive correlation between happiness and socioeconomic status.

\subsection{The Cultural and Social Context of Asian American Happiness}

Based on the findings of prior studies, one might expect Asian Americans to be happier than whites. Asian Americans have higher levels of education and income. Higher levels of education and income are associated with a higher level of overall life satisfaction. Therefore Asian Americans should have a higher level of happiness.

This hypothesized bivariate pattern—that Asian Americans are happier than whites—might not be observed in the population as a whole, however, due to confounding variables. As the result of more recent immigration patterns, Asian Americans tend to be younger. They are also more likely to be married. As these latter variables are associated with overall life satisfaction (Argyle, 1999), multivariate analysis is needed in order to ascertain whether Asian Americans systematically differ from whites.

Another important confounding factor is immigration. Asian Americans are likely to be foreign born immigrants (Sakamoto, Goyette, \& Kim, 2009). As immigrants, they encounter greater cultural conflicts, and moving to a new country is generally stressful. Language barriers, social isolation, misunderstandings, new expectations and roles, nostalgia for one’s country of origin, and identity crises may arise (Sue \& Morishima, 1982; Bhugra, 2004). Because Asian Americans are much more likely to be foreign born, this characteristic of being an immi- 
grant may reduce the level of happiness among Asian Americans relative to whites (who are overwhelmingly native born in the U.S.).

To a lesser extent, the impact of cultural differences may partially carry over to affect the level of happiness among second-generation Asian Americans. Native-born, second-generation Asian Americans often encounter some traditional Asian values and expectations at home but more liberal American norms among their peers, secondary groups, and in the media. This "acculturation discrepancy" (Kim, Chen, Wang, Shen, \& OrozcoLapray, 2013) is especially pronounced for Asian Americans because Asian cultures emphasize collectivist values while American society contrarily emphasizes individualistic values (Diener \& Suh, 1999; Markus \& Kitayama, 1991). Conflict between first-generation and second-generation Asian Americans is thus an old theme (Sakamoto, Goyette, \& Kim, 2009).

A common cultural difference is the larger parental investment in their children's future socioeconomic status and educational attainment among Asian parents than among white American parents (Sun, 1998; Sakamoto, Kim, \& Takei, 2012). Asian American children typically spend more time and effort involved in schooling and educational activities (Mau, 1997; Ainsworth-Darnell \& Downey, 1998; Sun, 1998; Jiménez \& Horowitz, 2013; Hsin \& Xie, 2014) than white children in part due to the higher educational expectations that Asian parents have for their children (Goyette \& Xie, 1999). Higher levels of parental investments and aspirations for their children's education are symptomatic of the more codependent and collectivist nature of Asian American families (Fuller, Holloway, Azuma, Hess, \& Kashiwagi, 1986; Sakamoto, Kim, \& Takei, 2012).

An extreme but journalistically popular example is provided by Amy Chua's (2011) personal memoir entitled The Battle Hymn of the Tiger Mom. This book describes the harsh parenting style of a stereotypically authoritarian Chinese American family. The mother was engaged in an almost ruthless compulsion to foster the superior educational achievements of her daughters (both of whom were later admitted to extremely prestigious universities). The book contends that a highly authoritarian parenting style (i.e., at least by mainstream American standards) helps to explain the notably higher educational attainments of Asian Americans. Similarly, the recent autobiography of the world's most famous concert pianist (i.e., Lang Lang's Journey of a Thousand Miles) describes the harsh parenting practices of a "Tiger Dad."

In terms of statistical evidence from nationally representative survey data, the higher educational attainments of Asian Americans is indeed a well-established finding that has been consistently evident for decades (Sakamoto, Goyette, \& Kim, 2009). The educational attainments of Asian Americans are greater than expected based on the social class origins of Asian Americans (e.g., even some working-class Asian Americans get accepted by prestigious universities). Asian American educational attainment is thus not simply derived from class-based socioeconomic advantages but is partly cultural in origin at least among second-generation Asian Americans (Kao, 1995; Sakamoto, Goyette, \& Kim, 2009; Hsin \& Xie, 2014). Although unusual cases to some degree, The Battle Hymn of the Tiger Mom and Journey of a Thousand Miles are nonetheless consistent with systematic statistical evidence on higher levels of Asian American educational achievement, and they also illustrate the greater educational investments and expectations that Asian parents often have for their children. ${ }^{1}$

Given this cultural context, Asian American children may grow up to become adults who are less happy than whites with equivalent levels of educational attainment. Second-generation Asian American adults may be more likely to be unhappy if they have strained social relations with their parents or have had a psychologically depressing (though not necessarily economically deprived) childhood (Kim, Chen, Wang, Shen, \& Orozco-Lapray, 2013). As noted earlier, conflict between first-generation and second-generation Asian Americans is seen as being common and is often related to the high educational expectations of Asian American immigrant parents. Second-generation Asian American adults may harper resentments towards their immigrant parents if they were too harsh by the standards of mainstream America (given the more lax childrearing practices of the U.S. relative to Asia). Second-generation Asian American adults may furthermore feel that they never fully satisfied the often unappeasable educational and career expectations of their demanding parents. ${ }^{2}$

In addition to conflict with parents over their high expectations, second-generation Asian American adults may be less likely to be happy if, due to family pressures, they were coerced into entering more financially lucrative careers rather than pursuing those fields of study or occupations that were more intrinsically appealing.

\footnotetext{
${ }^{1}$ Asian American parenting practices probably involve both authoritarian and authoritative styles and hopefully are not usually as obsessively negative as portrayed in The Battle Hymn of the Tiger Mom (Choi, Kim, Kim, \& Park, 2013).

${ }^{2}$ Much anecdotal evidence corroborates the findings of Jiménez and Horowitz’s (2013) ethnographic study characterizing a B grade on an exam as constituting an “Asian fail” (Jiménez \& Horowitz, 2013: p. 859).
} 
The expected financial returns to educational attainment are a common theme in the motivations of Asian American parents when they seek to influence their children's educational careers (Goyette \& Xie, 1999; Jiménez \& Horowitz, 2013). For example, prior research has shown that Asian Americans are far more likely than whites to enter into science, technology, engineering, and math (STEM) fields of study and occupations (Kim \& Sakamoto, 2010). The latter more consistently provide for a higher and more stable long-term income but many Americans apparently seem to find them to lead to less intrinsically rewarding jobs (Joyner, 2011).

A third possibility relates to personality in regard to the psychology of self-satisfaction. To some degree, many second-generation Asian Americans may have somewhat internalized their parents' tendencies to be more demanding of ever higher achievement. This insatiability leads to a higher rate of what psychologists refer to as hedonic adaptation (Frederick \& Loewenstein, 1999) or what sociologists have more generally described as the Veblen effect (Mirowsky, 1987). Having obtained a high level of education and income, second-generation Asian Americans may soon become accustomed (or "adapted") to accepting that achievement and then to no longer feel fully satisfied with it. More so than whites, second-generation Asian Americans may thus have ever increasing expectations for even higher achievements (due to having been raised by immigrant Asian American parents). This sort of internalized ambition is precisely the sort of personality trait that helped second-generation Asian Americans to become highly competitive in the first place.

Finally, a fourth possibility is racial discrimination of some sort. The level of Asian American happiness might be lower than that for whites if Asian Americans encounter racial discrimination or multicultural insensitivity. Whether the latter is blatantly "real” or only primarily "perceived," either type of experience could possibly generate a sense of resentment that could lead to the development of lower levels of happiness among Asian Americans compared to whites with similar socioeconomic status.

As noted earlier, empirically evaluating the importance of these hypothesized processes is beyond the scope of our data which are not primarily cultural or psychological in their orientation. Nonetheless, these proposed mechanisms are useful interpretations to consider in order to enhance our understanding of the possibility of complex relationships between socioeconomic characteristics and the level of happiness. These interpretations help to clarify the cultural and social context of Asian American happiness.

\section{Research Methods}

\subsection{Data and Variables}

We use data from the U.S. General Social Survey (GSS). This is a well-known and widely used cross-sectional survey that is nationally representative of the non-institutionalized adult population aged 18 and older in the U.S. It has been conducted annually for many years. We pool the annual surveys from 1972 to 2010 in order to obtain a large sample size with enough Asian Americans to permit multivariate analysis.

The data that we study are the responses to the survey question which is stated on the GSS as follows: "Taken all together, how would you say things are these days - would you say that you are very happy, pretty happy, or not too happy?” This question has a high response rate (Easterlin, 2001: p. 466). Many prior studies have investigated these data on happiness (e.g., Easterlin, 2001; Blanchflower \& Oswald, 2004; Kahneman \& Krueger, 2006) although without specifically considering Asian Americans.

Kahneman and Krueger (2006: pp. 7-9) review findings that provide some support for the validity of survey measures of subjective well-being. These studies find that the responses to this sort of survey question correlate with objective physiological and health measures, emotional conditions as revealed in neurological studies, various indicators of life circumstances, and ratings of one's happiness made by friends. According to Diener and Suh (1999: p. 437), results from survey questions on subjective well-being are generally not highly influenced by people's "propensity to be humble" or by issues relating to "language translation."

As mentioned above, we pool the annual surveys from 1972 to 2010 in order to increase the sample size of Asian Americans. In doing so, we obtain a sample size of 521 foreign-born Asian Americans and 137 nativeborn Asian Americans with observed data on happiness. While these figures do not constitute huge sample sizes, they are generally adequate for basic multivariate analysis. We employ standard statistical methods since these data can be treated as a representative sample over this time period.

Nonetheless, we acknowledge that 521 foreign-born Asian Americans plus 137 native-born Asian Americans implies that our total sample size for Asian Americans is just 658 which is less than 2 percent of the total sample and admittedly rather modest for a period spanning 4 decades. Unfortunately, this sample size is not sufficient to 
make highly detailed multivariate analysis for Asian Americans separately. In our approach, we simply include Asian American as a dichotomous variable in the regression models that also control for period effects over these decades. In this case where the estimates for the control variables will be dominated by the values associated with the much larger sample of whites, the statistical results for the Asian American coefficient are akin to standardization methods used in demographic analysis for smaller populations. Furthermore, this time period refers to the post-Civil Rights era in which the labor market circumstances and social attitudes towards Asian Americans were relatively stable compared to the pre-Civil Rights period (Sakamoto, Goyette, \& Kim, 2009). In short, these data do provide important empirical evidence that is better to be considered than to be ignored.

We limit our sample to persons aged 25 and older so as to avoid the volatile happiness levels of young people who are making the difficult life transition to adulthood (often fluctuating substantially in the short term). In order to provide some comparative findings that include some contrasts that are informative for understanding the net effects of being Asian American, we define the following race/ethnicity/nativity groups: 1) native born, non-Hispanic whites; 2) foreign born, non-Hispanic whites; 3) native born, non-Hispanic African Americans; (4) foreign born Asian Americans; and 5) native born Asian Americans. While other groups obviously could in principle be considered, our primary focus is on Asian Americans as we have discussed earlier. Space constraints and highly limited sample sizes prevent us from investigating other groups such as Native Americans.

As mentioned above, our approach utilizes multivariate analysis including various control variables. Age is measured in terms of five-year categories beginning with the ages 25 to 29. In order to account for period effects (i.e., happiness may be influenced by unmeasured conditions that change over time at the societal level), we include variables to indicate the interview time period including: 1972 to 1980; 1982 to 1986; 1987 to 1991; 1993 to 2000; and 2002 to 2010. Other categorical indicators include gender (i.e., whether female), marital status (i.e., currently married, never married, widowed, divorced/separated), and region of residence (i.e., the standard U.S. Census grouping of states into New England, Middle Atlantic, East North Central, West North Central, South Atlantic, East South Central, West South Central, Mountain, Pacific).

Education is measured in terms of the number of years completed as traditionally associated with each schooling level (i.e., high school graduation being 12 and a bachelor's degree being 16, and so forth). Number of children is an interval level variable (i.e., $0,1,2$, etc.) that refers to the total number of children that one has "ever had." Household income refers to the total income of related individuals residing together in the same living quarters in a given year of the survey (or individual income in the case of persons living alone). We measure income in terms of which quartile (i.e., bottom, just-below-the-median, just-above-the-median, top) that it is located in based on the distribution for each year considered separately.

To obtain our actual sample, we employ listwise deletion for all of the independent variables except income. Because income is a particularly important variable given our substantive interests, we do not wish to risk creating any inadvertent sample selection bias that might arise in the investigation of our research objectives. We therefore created a separate dichotomous independent variable (known as "Missing" for the income categories) that is equal to unity for cases that are missing on income (and zero otherwise). For similar reasons, we do not delete cases that are "don’t know" or missing on the happiness variable (i.e., our dependent variable) but instead define them to constitute a separate outcome category that is empirically studied.

\subsection{Statistical Models}

For our multivariate statistical analysis we estimate multinomial logistic regression models. Because the main outcomes for our dependent variable are ordered categories, an ordered logistic regression might seem to be a more straightforward approach. However, the latter model includes a multiplicative constraint which assumes that the net effect of an independent variable on the chances of going from "not to happy" to "pretty happy" is proportional to the chances of going from "pretty happy" to "very happy." Although that assumption is convenient in that it reduces the number of parameters to be estimated, preliminary analysis indicated that the net effects of many independent variables are not proportional in this way as assumed by the ordered logistic model. We therefore use the multinomial logistic model which does not make any such assumptions of proportionality. Our findings are thereby more robust. ${ }^{3}$

\footnotetext{
${ }^{3}$ Multinomial logistic regression furthermore allows us to include “missing on happiness" as a separate categorical outcome in itself. Our models do include this nonresponse as an outcome variable. We do not show these results in the tables because the estimates are mostly not significant and do not alter any of our main conclusions.
} 


\section{Empirical Results}

\subsection{Descriptive Statistics}

As shown at the bottom of Table 1, the total sample size is 37,323 including 30,170 native born, non-Hispanic whites (hereafter, “whites”), 1418 foreign born, non-Hispanic whites (hereafter, “foreign born whites”), 5077 native born, non-Hispanic African Americans (hereafter, "blacks"), 512 foreign born Asian Americans (hereafter, "foreign born Asians"), and 137 native born Asian Americans (hereafter, "native born Asians"). Although whites are overwhelmingly the largest majority group (constituting $81 \%$ of our total sample as shown in Table 1), that percentage accurately reflects the population composition in the U.S. for this overall time period given that our sample includes data going back to $1972 .^{4}$

Table 1. Descriptive statistics by race/ethnicity and nativity, general social survey 1972-2010.

\begin{tabular}{|c|c|c|c|c|c|c|c|}
\hline & Full sample & $\begin{array}{l}\text { US-born } \\
\text { NH-White }\end{array}$ & $\begin{array}{l}\text { Foreign-born } \\
\text { NH-White }\end{array}$ & $\begin{array}{l}\text { US-born } \\
\text { NH-Black }\end{array}$ & $\begin{array}{l}\text { Foreign-born } \\
\text { Asian }\end{array}$ & $\begin{array}{l}\text { US-born } \\
\text { Asian }\end{array}$ & $\begin{array}{c}\text { Asian } \\
\text { Combined }\end{array}$ \\
\hline \multicolumn{8}{|l|}{ Happiness } \\
\hline Very happy & 0.30 & 0.31 & 0.29 & 0.21 & 0.29 & 0.20 & 0.27 \\
\hline Pretty happy & 0.53 & 0.53 & 0.51 & 0.53 & 0.48 & 0.58 & 0.50 \\
\hline Not too happy & 0.11 & 0.10 & 0.12 & 0.18 & 0.13 & 0.09 & 0.12 \\
\hline Missing & 0.07 & 0.07 & 0.08 & 0.08 & 0.11 & 0.13 & 0.11 \\
\hline (Total) & $(1.00)$ & $(1.00)$ & $(1.00)$ & $(1.00)$ & $(1.00)$ & $(1.00)$ & $(1.00)$ \\
\hline US-born NH-White & 0.81 & & & & & & \\
\hline Foreign-born NH-White & 0.04 & & & & & & \\
\hline US-born NH-Black & 0.14 & & & & & & \\
\hline Foreign-born Asian & 0.01 & & & & & & \\
\hline US-born Asian & 0.004 & & & & & & 0.79 \\
\hline Age & & & & & & & 0.21 \\
\hline $25-29$ & 0.12 & 0.11 & 0.09 & 0.14 & 0.15 & 0.22 & 0.16 \\
\hline $30-34$ & 0.12 & 0.12 & 0.10 & 0.14 & 0.21 & 0.18 & 0.20 \\
\hline $35-39$ & 0.12 & 0.12 & 0.11 & 0.12 & 0.17 & 0.07 & 0.15 \\
\hline $40-44$ & 0.11 & 0.11 & 0.11 & 0.11 & 0.14 & 0.11 & 0.13 \\
\hline $45-49$ & 0.10 & 0.10 & 0.09 & 0.09 & 0.09 & 0.08 & 0.09 \\
\hline $50-54$ & 0.09 & 0.09 & 0.08 & 0.09 & 0.08 & 0.05 & 0.07 \\
\hline $55-59$ & 0.08 & 0.08 & 0.08 & 0.08 & 0.06 & 0.12 & 0.07 \\
\hline $60-64$ & 0.07 & 0.07 & 0.06 & 0.06 & 0.04 & 0.05 & 0.04 \\
\hline $65-69$ & 0.07 & 0.07 & 0.07 & 0.06 & 0.02 & 0.04 & 0.03 \\
\hline $70-74$ & 0.06 & 0.06 & 0.07 & 0.04 & 0.02 & 0.03 & 0.02 \\
\hline $75-79$ & 0.04 & 0.04 & 0.06 & 0.03 & 0.02 & 0.01 & 0.02 \\
\hline $80-84$ & 0.03 & 0.03 & 0.05 & 0.01 & 0.004 & 0.02 & 0.01 \\
\hline 85 - 89+ & 0.02 & 0.02 & 0.03 & 0.01 & 0.004 & 0.01 & 0.01 \\
\hline (Total) & $(1.00)$ & $(1.00)$ & $(1.00)$ & $(1.00)$ & $(1.00)$ & $(1.00)$ & $(1.00)$ \\
\hline \multicolumn{8}{|l|}{ Interview year } \\
\hline $1972-1980$ & 0.10 & 0.11 & 0.13 & 0.07 & 0.04 & 0.02 & 0.03 \\
\hline 1982-1986 & 0.18 & 0.18 & 0.18 & 0.18 & 0.07 & 0.09 & 0.08 \\
\hline
\end{tabular}




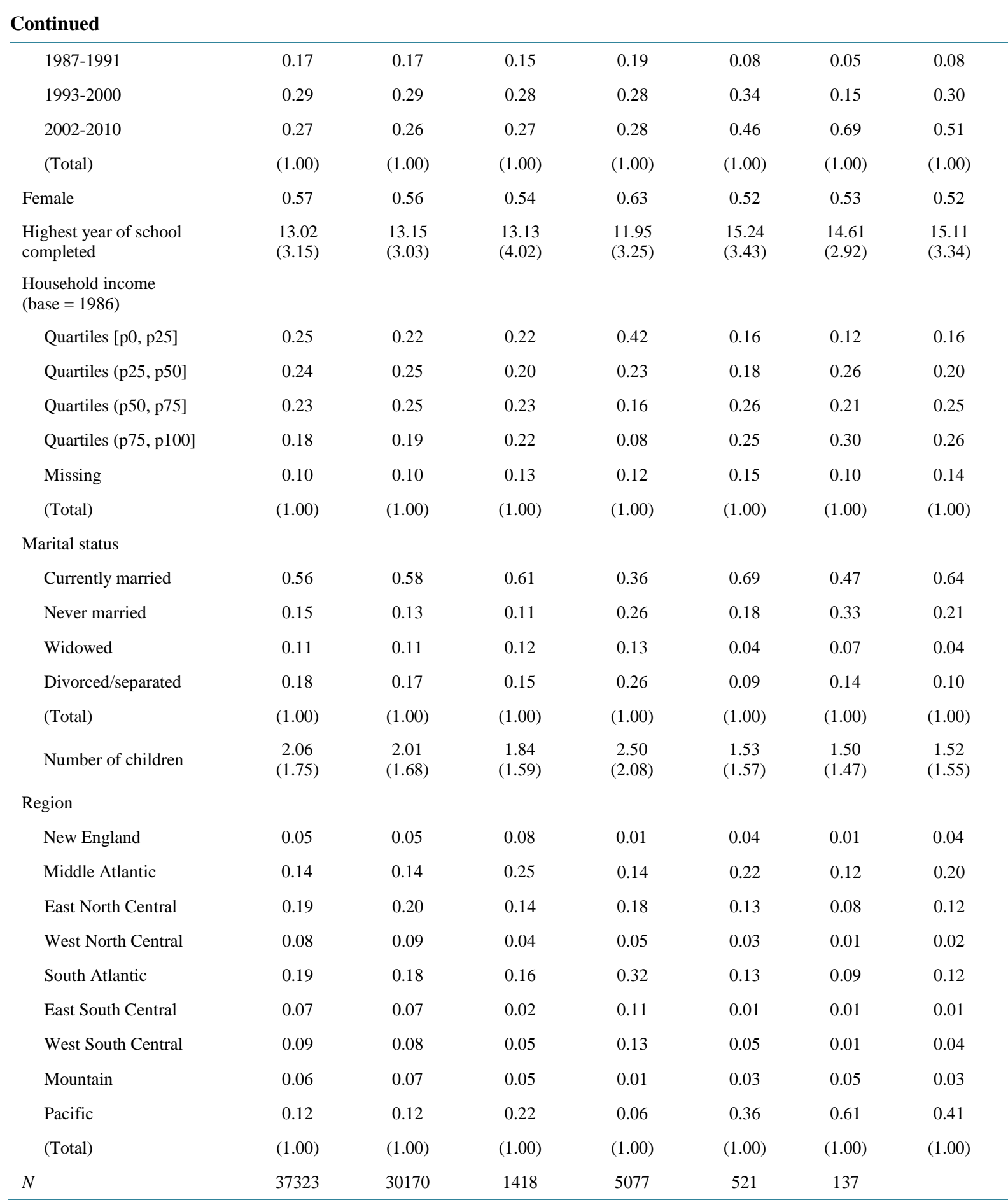

The smaller sample sizes for foreign born Asians and native born Asians implies that we cannot obtain very precise results for these two groups in regression models estimated separately for them. We attempted to do so but did not obtain many statistically significant findings using our primary model specification that includes a rather large number of independent variables many of which are fairly highly correlated. As mentioned above, our general approach for the multivariate analysis is therefore to combine all of the groups together into a single regression model in which dichotomous variables are included to indicate each of the minority race/ethnicity/ nativity groups using whites as the reference category. 
In regard to the dependent variable, Table 1 indicates that most of the sample is "pretty happy" (hereafter, "PH"), namely, 53\%. 30\% are "very happy" (hereafter, "VH") while just 11\% are "not too happy" (hereafter, "NTH") and 7\% are missing. Thus, most people report being PH while VH is next most common outcome with NTH being somewhat uncommon. The cases that are missing on happiness are disproportionately from the last decade of the data when nonresponse rates increased. Although Table 1 shows that Asian Americans are somewhat more likely to be missing on happiness than whites overall in these data, this pattern reflects the fact that the Asian American population had proportionately increased after 2002. Net of time period, Asian Americans are not more likely to be missing than whites. ${ }^{5}$

As for racial differentials, Table 1 shows that the outcomes for whites are close to the pattern for the entire sample because whites are the overwhelmingly majority of that sample. On the other hand, although constituting a rather small demographic group, foreign-born whites have a happiness distribution that is quite close to whites. The groups that more particularly stand out are blacks and native-born Asians who are less likely to be $\mathrm{VH}$, and blacks and foreign-born Asians who are more likely to be NTH.

Consistent with many other studies, Table 1 shows blacks have a lower mean years of schooling than whites while foreign-born Asians and native-born Asians have higher means. Foreign-born whites have a mean years of schooling that is nearly identical to whites. Also consistent with prior research, foreign-born Asians and native-born Asians are more likely to be in the upper quartile of the distribution of household income in any given year while blacks are more likely to be in the bottom quartile (Sakamoto, Goyette, \& Kim, 2009).

In regard to number of children, blacks have the highest mean while foreign-born whites, foreign-born Asians, and native-born Asians have means that are slightly lower than whites. Blacks are disproportionately located in the southern areas while whites are disproportionately located in the north central areas. Foreign-born Asians and especially native-born Asians are disproportionately located in the Pacific. Foreign-born Asians and foreign-born whites are also slightly more likely to be residents of the Middle Atlantic.

Table 2 shows the cross-classification of happiness by race/ethnicity/nativity group. This information is also contained in the first four rows of Table 1 and the most basic patterns were already discussed above. However, Table 2 more clearly shows that for each group, PH is the modal category while NTH is the least common reported happiness level.

\subsection{Multivariate Statistical Results}

These foregoing descriptive statistics are important in documenting the overall empirical contours of reported life satisfaction with respect to socioeconomic and demographic variables including race and ethnicity. However, as discussed earlier, our more theoretical interest is to understand whether Asian Americans have the same levels of happiness as whites after controlling for the higher levels of education and incomes of Asian Americans. In order to assess the robustness of our empirical conclusions, we also report results for slightly varying specifications that also include additional control variables.

Table 2. Cross-tabulations of overall life satisfaction by race/ethnicity and nativity.

\begin{tabular}{|c|c|c|c|c|c|}
\hline Race-ethnicity-nativity & Very happy & Pretty happy & Not too happy & Missing & Total \\
\hline US-born non-Hispanic White & $\begin{array}{c}9473 \\
(31.4 \%)\end{array}$ & $\begin{array}{c}15,846 \\
(52.52 \%)\end{array}$ & $\begin{array}{c}2875 \\
(9.53 \%)\end{array}$ & $\begin{array}{c}1976 \\
(6.55 \%)\end{array}$ & $\begin{array}{l}30,170 \\
(100 \%)\end{array}$ \\
\hline Foreign-born non-Hispanic White & $\begin{array}{c}415 \\
(29.27 \%)\end{array}$ & $\begin{array}{c}723 \\
(50.99 \%)\end{array}$ & $\begin{array}{c}169 \\
(11.92 \%)\end{array}$ & $\begin{array}{c}111 \\
(7.83 \%)\end{array}$ & $\begin{array}{c}1,418 \\
(100 \%)\end{array}$ \\
\hline US-born non-Hispanic Black & $\begin{array}{c}1059 \\
(20.86 \%)\end{array}$ & $\begin{array}{c}2709 \\
(53.36 \%)\end{array}$ & $\begin{array}{c}919 \\
(18.1 \%)\end{array}$ & $\begin{array}{c}390 \\
(7.68 \%)\end{array}$ & $\begin{array}{c}5077 \\
(100 \%)\end{array}$ \\
\hline Foreign-born Asian & $\begin{array}{c}149 \\
(28.6 \%)\end{array}$ & $\begin{array}{c}249 \\
(47.79 \%)\end{array}$ & $\begin{array}{c}67 \\
(12.86 \%)\end{array}$ & $\begin{array}{c}56 \\
(10.75 \%)\end{array}$ & $\begin{array}{c}521 \\
(100 \%)\end{array}$ \\
\hline US-born Asian & $\begin{array}{c}28 \\
(20.44 \%)\end{array}$ & $\begin{array}{c}79 \\
(57.66 \%)\end{array}$ & $\begin{array}{c}12 \\
(8.76 \%)\end{array}$ & $\begin{array}{c}18 \\
(13.14 \%)\end{array}$ & $\begin{array}{c}137 \\
(100 \%)\end{array}$ \\
\hline Total & $\begin{array}{c}11,124 \\
(29.8 \%)\end{array}$ & $\begin{array}{c}19,606 \\
(52.53 \%)\end{array}$ & $\begin{array}{c}4042 \\
(10.83 \%)\end{array}$ & $\begin{array}{c}2551 \\
(6.83 \%)\end{array}$ & $\begin{array}{c}37,323 \\
(100 \%)\end{array}$ \\
\hline
\end{tabular}

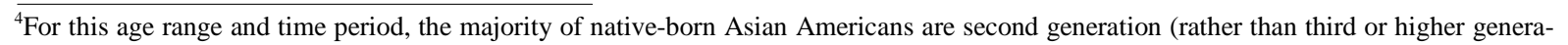
tion) particularly since our analysis is restricted to “single race” persons (Sakamoto, Goyette, \& Kim, 2009).

${ }^{5}$ These and other multivariate results for missing as an outcome on happiness are available upon request. 
Because these GSS data are derived from cross-sectional surveys, none of these variables has any clear longitudinal aspect relative to the time that the dependent variable was measured. Therefore, these data do not naturally provide any clear statistical basis to assume that certain covariates are fully exogenous with respect to happiness. For example, we cannot conclusively rule out the possibility that some people are never married partly because they are intrinsically not too happy or that happier people move to the south (i.e., some reverse causation may be operating). Our general approach is to estimate various model specifications in order to see how sensitive the estimates are for the race/ethnicity/nativity variables. In general, we believe that age, gender, race/ ethnicity/nativity, and year of the interview are all strongly exogenous with respect to our measure of happiness. We also believe, based on prior research (Argyle, 1999; Easterlin, 2001; Kahneman \& Krueger, 2006), that education, income, and family structure are primarily exogenous with this sort of cross-sectional data, but we recognize that this is an interpretation.

Table 3 reports the estimates for the prediction of being VH (relative to being PH) while Table 4 reports the estimates for the prediction of being NTH (relative to being PH). As mentioned above, we also estimated the regression of being missing on happiness (relative to being PH) so as to obtain more reliable results overall (because the statistical model optimizes all for the estimates for each of these contrasts simultaneously). We do not, however, show the results for this latter regression because they do not indicate any substantively significant or notable patterns (except that nonresponse became more common in the last decade of the data).

The different model specifications shown in Table 3 and Table 4 provide the reader with greater insight and detail about how sensitive the net effects of each race/ethnicity/nativity group are given different sets of control variables. Model 2 controls for age and interview year. The coefficients for the latter suggest that, in regard to being $\mathrm{VH}$ as shown in Table 3, the chances of being VH appear to be declining slightly over this time period. Because more native born Asians are entering into adulthood in the later years of our survey data (i.e., most native born Asians were born sometime after the 1965 Immigration Act and so as adults they are disproportionately to be found in the data for the later 1990's and 2000's) and because the chances of being VH seem to be declining slightly over time in American society, the net effect of being native born Asian in Model 3 is -0.460 which is more attenuated (i.e., closer to zero) than in Model 1 where the estimate is -0.523 without controlling for age and interview year. The bivariate estimate of -0.523 partly incorporates the effect of interview year (as well as age) rather than representing only the effect of being native born Asian per se.

Table 3. Multinomial logistic regression of being very happy (pretty happy as reference, $\mathrm{N}=37,323$ ).

\begin{tabular}{|c|c|c|c|c|c|c|c|}
\hline Variable & Model 1 & Model 2 & Model 3 & Model 4 & Model 5 & Model 6 & Model 7 \\
\hline \multicolumn{8}{|l|}{ Race-ethnicity-nativity (ref. = NB-NH-W) } \\
\hline Foreign-born non-Hispanic White & -0.041 & -0.055 & -0.054 & -0.06 & -0.086 & -0.106 & -0.076 \\
\hline US-born non-Hispanic Black & $-0.425^{* * *}$ & $-0.403^{* * *}$ & $-0.407^{* * *}$ & $-0.362^{* * *}$ & $-0.268^{* * *}$ & $-0.170^{* * *}$ & $-0.207^{* * *}$ \\
\hline Foreign-born Asian & 0.001 & 0.078 & 0.079 & 0.031 & 0.032 & -0.047 & -0.023 \\
\hline US-born Asian & $-0.523^{*}$ & $-0.460^{*}$ & $-0.459^{*}$ & $-0.487^{*}$ & $-0.549^{*}$ & $-0.471^{*}$ & $-0.457^{*}$ \\
\hline Age & & Yes & Yes & Yes & Yes & Yes & Yes \\
\hline Interview year & & Yes & Yes & Yes & Yes & Yes & Yes \\
\hline Female & & & Yes & Yes & Yes & Yes & Yes \\
\hline Highest year of school completed & & & & Yes & Yes & Yes & Yes \\
\hline Marital Status & & & & & & Yes & Yes \\
\hline Number of children & & & & & & Yes & Yes \\
\hline Interaction of female and \# of children & & & & & & Yes & Yes \\
\hline Region & & & & & & & Yes \\
\hline Constant & $-0.514^{* * *}$ & $-0.501^{* * *}$ & $-0.524^{* * *}$ & $-0.949^{* * *}$ & $-0.968^{* * *}$ & $-0.748^{* * *}$ & $-0.892^{* * *}$ \\
\hline
\end{tabular}

${ }^{* * *} p<0.001,{ }^{* *} p<0.01, \stackrel{*}{p}<0.05,{ }^{+} p<0.10$. 
Table 4. Multinomial logistic regression of being not too happy (pretty happy as reference, $N=37,323$ ).

\begin{tabular}{|c|c|c|c|c|c|c|c|}
\hline Variable & Model 1 & Model 2 & Model 3 & Model 4 & Model 5 & Model 6 & Model 7 \\
\hline \multicolumn{8}{|c|}{ Race-ethnicity-nativity (ref. = NB-NH-W) } \\
\hline Foreign-born non-Hispanic white & $0.253^{* *}$ & $0.226^{*}$ & $0.227^{*}$ & $0.218^{*}$ & $0.253^{* *}$ & $0.276^{* *}$ & $0.229^{*}$ \\
\hline US-born non-Hispanic black & $0.626^{* * *}$ & $0.652^{* * *}$ & $0.651^{* * *}$ & $0.536^{* * *}$ & $0.402^{* * *}$ & $0.329^{* * *}$ & $0.323^{* * *}$ \\
\hline Foreign-born Asian & $0.394^{* *}$ & $0.455^{* *}$ & $0.456^{* *}$ & $0.547^{* * *}$ & $0.542^{* * *}$ & $0.639^{* * * *}$ & $0.576^{* * *}$ \\
\hline US-born Asian & -0.178 & -0.176 & -0.176 & -0.115 & -0.02 & -0.076 & -0.158 \\
\hline Age & & Yes & Yes & Yes & Yes & Yes & Yes \\
\hline Interview year & & Yes & Yes & Yes & Yes & Yes & Yes \\
\hline Female & & & Yes & Yes & Yes & Yes & Yes \\
\hline Highest year of school completed & & & & Yes & Yes & Yes & Yes \\
\hline Household income & & & & & Yes & Yes & Yes \\
\hline Marital Status & & & & & & Yes & Yes \\
\hline Number of children & & & & & & Yes & Yes \\
\hline Interaction of female and \# of children & & & & & & Yes & Yes \\
\hline Region & & & & & & & Yes \\
\hline Constant & $-1.707^{* * *}$ & $-1.834^{* * *}$ & $-1.844^{* * *}$ & $-0.820^{* * *}$ & $-0.798^{* * *}$ & $-1.113^{* * *}$ & $-1.180^{* * *}$ \\
\hline
\end{tabular}

$p<0.001, \stackrel{* *}{p} p<0.01,{ }^{*} p<0.05,{ }^{+} p<0.10$.

In regard to coefficients from logistic regression, they refer to effects in terms of logged odds ratios. Taking the anti-log of -0.460 and subtracting from unity yields the multiplicative effect in terms of the odds ratio itself. Thus, exp $(-0.460)-1=-0.37$ implies that the odds ratio of being $\mathrm{VH}$ (relative to being $\mathrm{PH}$ ) declines by $37 \%$ for native-born Asians controlling for age and interview year. This result is statistically significant at the 0.05 level as indicated by its single asterisk as shown for Model 2 in Table 3.

Other results from Model 2 indicate that the coefficients for foreign-born Asians and for foreign-born whites are not statistically significant. Indeed, these coefficients are not statistically significant in any of the model specifications in Table 3. These results indicate that foreign-born Asians and foreign-born whites do not differ from whites in terms of the chances for being VH. The group that does have a statistically significant effect (in addition to native-born Asians) is blacks. Their coefficient is negative and statistically significant in each of the models in Table 3. Although not as large (in absolute value) as the effects for native-born Asians, the estimates for blacks indicate that they have lower chances of being VH compared to whites.

The full specification is Model 7 which uses all of the control variables including age, interview year, gender, education, income, marital status, number of children, and region of residence. Table 3 shows that the coefficient for native born Asians in Model 7 is -0.457 which is statistically significant. This finding again indicates $37 \%$ lower odds of being VH for this group after accounting for all of the control variables. For blacks, the coefficient in Model 7 is -0.207 which is statistically significant and implies that blacks have $19 \%$ lower odds of being $\mathrm{VH}$ net of the control variables.

Table 4 shows the results for the same model specifications except that the outcome is being NTH (relative to being PH). The results for the bivariate specification are shown in Model 1 in Table 4. The coefficients for each of the race/ethnicity/nativity group are statistically significant except for native-born Asians. For foreign-born whites, the coefficient is 0.253 which implies that this group has $29 \%$ higher odds (i.e., exp $(0.253)-1=29 \%$ ) of being NTH compared to whites. For blacks, the coefficient is 0.626 which implies that this group has $87 \%$ higher odds (i.e., exp (0.626) $-1=87 \%$ ) of being NTH compared to whites. For foreign-born Asians, the coefficient is 0.394 which implies that this group has $48 \%$ higher odds (i.e., exp $(0.394)-1=48 \%$ ) of being NTH compared to whites.

In Model 2 which controls for age and interview year, the coefficient that changes the most is for foreign-born Asians which increases to 0.455 and continues to be statistically significant. In the full specification which in- 
cludes all of the control variables (i.e., Model 7) the coefficient for foreign born Asians increases still further to 0.576 which implies that this group has $78 \%$ higher odds (i.e., exp (0.576) $-1=78 \%$ ) of being NTH compared to whites. While the net effect of being a foreign-born Asian thus increases after controlling for age, interview year, gender, education, income, marital status, number of children, and region of residence, the coefficient for blacks conversely decreases to 0.323 in Model 7 (indicating that blacks have 38\% higher odds of being NTH compared to whites). Also in contrast to foreign-born Asians, the coefficient for foreign-born whites in Model 7 does not change very much relative to estimate from Model 1 while the coefficient for native-born Asians continues to be not statistically significant in Model 7.

\section{Discussion}

To our knowledge, this study is the first to investigate survey data on the overall life satisfaction of Asian Americans. Our general approach has been fairly exploratory due to a lack of prior research on this topic for this group. Our results indicate that foreign-born Asians and native-born Asians are not happier than whites despite the higher levels of education and income among these two Asian groups. While the proportion of whites who are NTH is a tiny bit higher than for native-born Asians in Table 2, the coefficient for native-born Asians in Model 1 for being NTH in Table 4 (i.e., the bivariate specification as represented in the logistic regression corresponding to the bivariate results as obtained in the cross-tabulation shown in Table 3) implies that this slight racial difference in Table 2 is not statistically significant.

However, the coefficient for foreign-born Asians in Model 1 for being NTH in Table 4 is substantively large and highly significant as is the (negative) coefficient for native-born Asians in Model 1 for being VH in Table 3. Foreign-born Asians are thus more likely to be NTH while native-born Asians are less likely to be VH. These patterns still hold substantially as well in the results from Model 2 which controls for age and interview year. Thus, there is no clear evidence that either Asian group is ever happier than whites but there is consistent evidence that Asians are either more likely to be less happy or less likely to be more happy. This pattern arises despite the fact that, when compared to whites, both foreign born Asians and native born Asians are more likely to have more education and to be in the top quartile of the income distribution.

In terms of the multivariate results based on the full specification that includes all of the control variables such as education and income (i.e., Model 7), the negative effects for the Asian groups persist. For native-born Asians, their lower chances of being VH are attenuated only slightly after taking into account their higher levels of education and income compared to whites (i.e., Table 3). Although African Americans are also less likely than whites to be VH according to the full specification (i.e., Model 7 in Table 3), blacks' chances are not as negative as those for native-born Asians. Furthermore, about half of the negative chances for blacks are statistically explained by the control variables whereas the negative chances for native-born Asians are only slightly explained by the control variables.

For foreign-born Asians, their higher chances of being NTH are slightly increased after taking into account their higher levels of education and income compared to whites (i.e., Models 6 and 7 in Table 4). Although blacks also have higher chances than whites of being NTH in the full specification (i.e., Models 6 and 7 in Table 4), the higher chances among blacks are more substantially explained (and they are certainly not increased) after taking into account the control variables. While only about $11 \%$ of the overall sample reports being NTH (i.e., that is clearly not the modal outcome) foreign-born Asians appear to be much more susceptible to this characteristic. To some extent this outcome may be more likely among immigrants who are uprooted from their countries of origin since even foreign-born whites are also more likely to be NTH after controlling for the other variables (i.e., Model 7 in Table 4). However, the chances for foreign-born Asians to be NTH are greater than for foreign-born whites or African Americans.

\section{Conclusion}

Earlier we discussed several possible sources of lower overall life satisfaction among Asian Americans. While our empirical analysis cannot definitely draw strong conclusions about specific causal processes, it does suggest certain interpretations. We hope that our initial investigation of happiness among Asian Americans will stimulate future research on this topic.

Although foreign-born whites are more likely to be NTH compared to native-born whites, foreign-born Asians are still slightly more likely to be NTH compared to foreign-born whites. This differential suggests that 
the acculturation process may be more difficult for Asians than for whites. Foreign-born Asians may find it harder to establish reliable networks of social support due to greater language barriers or cultural differences. Foreign-born Asians may also be particularly susceptible to psychological tensions associated with cultural conflict given the greater collectivist values of Asian societies that contrast with the highly individualistic assumptions of contemporary American culture.

An additional social psychological consideration is that Asians may also simply be more critical of their own selves which may help to propel them to higher levels of achievement and greater competitiveness but which may simultaneously make them less happy with what they have (Ng, 2002). This latter factor is perhaps suggested by the models in Table 4 because the negative net effect of being a foreign-born Asian becomes even more negative in those models that control for education and income.

Racial discrimination may be an additional factor affecting the happiness levels of Asian Americans and our findings cannot rule out its significance given that we have no direct measure of it. Nonetheless, we are somewhat reluctant to consider it to be a primary factor at least as a direct causal process. After controlling for education and income, the negative effects for the two Asian groups are greater (i.e., more negative) than for African Americans. Given the continued higher levels of residential segregation and lower levels of intermarriage between whites and blacks, we find it difficult to believe that negative attitudes are worse towards Asian Americans than towards African Americans. Discrimination is likely greater towards African Americans and yet our results show less negative effects for them relative to Asian Americans after controlling for education and income.

In regard specifically to native-born Asians, their situation is a bit different from foreign-born Asians because the pattern for the native born is not the higher chances of being NTH but the lower chances of being VH. Future research may focus more specifically on whether this finding may in part derive from specific patterns of educational and occupational attainments among native-born Asians (e.g., STEM fields). Lower levels of being VH may perhaps help to propel native-born Asian parents to become more acculturated, mainstream American parents themselves thereby enhancing the assimilation of Asian Americans. Accordingly, the statistical evidence indicates that educational attainment among specifically third-generation Asians tends to be lower than for second-generation Asians (Sakamoto, Goyette, \& Kim, 2009).

While the differential in VH for native-born Asians definitely indicates lower overall life satisfaction among them compared to native-born whites, it should be further noted that being $\mathrm{VH}$ is generally more uncommon than being PH in the population as a whole. Future research may also investigate more fully the possible effects of specific patterns of intermarriage, civic participation, religiosity, and the possibility of strained relations between adult offspring and their aging parents. Our results are at least noteworthy in pointing out that despite usually having foreign-born Asian parents (some of whom may possibly exhibit “Tiger Mom” tendencies), native-born Asian American adults do not appear to be more likely to be NTH than whites.

Our study is not without limitations. First, the measurement of overall life satisfaction is not precise since it is indicated as simply a categorical variable with three ordered outcomes. Future research might investigate data such as the World Values Survey which measures life satisfaction in terms of a numeric scale. Second, our sample size of Asian Americans is not especially large. Future research may seek to obtain additional data for Asian Americans by including the most current years of the U.S. General Social Survey or other data sets. Finally, if the sample size of Asian Americans can be significantly increased, then future research might be able to investigate ethnic differentials among Asian Americans (e.g., Chinese Americans versus Japanese Americans, etc.).

\section{References}

Ainsworth-Darnell, J. W., \& Downey, D. B. (1998). Assessing the Oppositional Culture Explanation for Racial/Ethnic Differences in School Performance. American Sociological Review, 63, 536-553. http://dx.doi.org/10.2307/2657266

Argyle, M. (1999). Causes and Correlates of Happiness. In D. Kahneman, E. Diener, \& N. Schwarz (Eds.), Well-Being: The Foundations of Hedonic Psychology (pp. 353-373). New York: Russell Sage Foundation.

Bhugra, D. (2004). Migration and Mental Health. Acta Psychiatrica Scandinavica, 109, 243-258. http://dx.doi.org/10.1046/j.0001-690X.2003.00246.x

Blanchflower, D. G., \& Oswald, A. J. (2004). Well-Being over Time in Britain and the USA. Journal of Public Economics, 88, 1359-1386. http://dx.doi.org/10.1016/S0047-2727(02)00168-8

Campbell, A., Converse, P. E., \& Rodgers, W. L. (1975). The Quality of American Life. New York: Sage. 
Choi, Y., Kim, Y. S., Kim, S. Y., \& Park, I. J. (2013). Is Asian American Parenting Controlling and Harsh? Empirical Testing of Relationships between Korean American and Western Parenting Measures. Asian American Journal of Psychology, 4, 19-29. http://dx.doi.org/10.1037/a0031220

Chua, A. (2011). The Battle Hymn of the Tiger Mother. New York: Penguin Press.

Clark, A. E., Frijters, P., \& Shields, M. A. (2008). Relative Income, Happiness, and Utility: An Explanation for the Easterlin Paradox and Other Puzzles. Journal of Economic Literature, 46, 95-144. http://dx.doi.org/10.1257/jel.46.1.95

Diener, E., \& Suh, E. M. (1999). National Differences in Subjective Well-Being. In D. Kahneman, E. Diener, \& N. Schwarz (Eds.), Well-Being: The Foundations of Hedonic Psychology (pp. 434-469). New York: Russell Sage Foundation.

Easterlin, R. A. (2001). Income and Happiness: Towards a Unified Theory. The Economic Journal, 111, 465-484. http://dx.doi.org/10.1111/1468-0297.00646

Frederick, S., \& Loewenstein, G. (1999). Hedonic Adaptation. In D. Kahneman, E. Diener, \& N. Schwarz (Eds.), Well-Being: The Foundations of Hedonic Psychology (pp. 302-329). New York: Russell Sage Foundation.

Fuller, B., Holloway, S., Azuma, H., Hess, R. D., \& Kashiwagi, K. (1986). Contrasting Achievement Rules: Socialization of Japanese School Children at Home and in School. Research in Sociology of Education and Socialization, 6, 165-201.

Goyette, K. A., \& Xie, Y. (1999). Educational Expectations of Asian American Youths: Determinants and Ethnic Differences. Sociology of Education, 72, 22-36. http://dx.doi.org/10.2307/2673184

Graham, C. (2009). Happiness around the World: The Paradox of Happy Peasants and Miserable Millionaires. New York: Oxford University Press. http://dx.doi.org/10.1093/acprof:osobl/9780199549054.001.0001

Hsin, A., \& Xie, Y. (2014). Explaining Asian Americans’ Academic Advantage over Whites. Proceedings of the National Academy of Sciences of the United States of America, 111, 8416-8421. http://dx.doi.org/10.1073/pnas.1406402111

Jiménez, T. R., \& Horowitz, A. (2013). When White Is Just Alright: How Immigrants Redefine Achievement and Reconfigure the Ethnoracial Hierarchy. American Sociological Review, 78, 849-871. http://dx.doi.org/10.1177/0003122413497012

Joyner, J. (2011). Why More Americans Don’t Major in Math and Science. Outside the Beltway, 9 November 2011. http://www.outsidethebeltway.com/why-more-americans-dont-major-in-the-math-and-science/

Kahneman, D., \& Krueger, A. B. (2006). Developments in the Measurement of Subjective Well-Being. Journal of Economic Literature, 20, 3-24. http://dx.doi.org/10.1257/089533006776526030

Kao, G. (1995). Asian-Americans as Model Minorities? A Look at their Academic Performance. American Journal of Education, 103, 121-159. http://dx.doi.org/10.1086/444094

Kim, C., \& Sakamoto, A. (2010). Have Asian American Men Achieved Labor Market Parity with White Men? American Sociological Review, 75, 934-957.

Kim, S. Y., Chen, Q., Wang, Y. J., Shen, Y. S., \& Orozco-Lapray, D. (2013). Longitudinal Linkages Among Parent-Child Acculturation Discrepancy, Parenting, Parent-Child Sense of Alienation, and Adloescent Adjustment in Chinese Immigrant Families. Developmental Psychology, 49, 900-912. http://dx.doi.org/10.1037/a0029169

Markus, H. R., \& Kitayama, S. (1991). Culture and the Self: Implications for Cognition, Emotion, and Motivation. Psychological Review, 98, 224-253. http://dx.doi.org/10.1037/0033-295X.98.2.224

Mau, W.-C. (1997). Parental Influences on High School Students’ Academic Achievement: A Comparison of Asian Immigrants, Asian Americans, and White Americans. Psychology in the Schools, 34, 267-277. http://dx.doi.org/10.1002/(SICI)1520-6807(199707)34:3<267::AID-PITS9>3.0.CO;2-L

Mirowsky, J. (1987). The Psycho-Economics of Feeling Underpaid: Distributive Justice and the Earnings of Husbands and Wives. American Journal of Sociology, 92, 1404-1434. http://dx.doi.org/10.1086/228670

Ng, Y. K. (2002). East-Asian Happiness Gap. Pacific Economic Review, 7, 51-63. http://dx.doi.org/10.1111/1468-0106.00149

Pew Research Center (2013). The Rise of Asian Americans. http://www.pewsocialtrends.org/2012/06/19/the-rise-of-asian-americans/

Ravallion, M., \& Lokshin, M. (2002). Self-Rated Economic Welfare in Russia. European Economic Review, 46, $1453-1473$. http://dx.doi.org/10.1016/S0014-2921(01)00151-9

Sakamoto, A., Kim, C., \& Takei, I. (2012). The Japanese-American Family. In R. Wright (Ed.), Ethnic Families in America (pp. 22-276). New York: Prentice Hall.

Sakamoto, A., Goyette, K. A., \& Kim, C. (2009). Socioeconomic Attainments of Asian Americans. Annual Review of Sociology, 35, 255-276. http://dx.doi.org/10.1146/annurev-soc-070308-115958

Sue, S., \& Morishima, J. K. (1982). The Mental Health of Asian Americans. San Francisco, CA: Jossey-Bass. 